\title{
On Approximate Pricing of Spread Options via Conditional Value-at-Risk
}

\author{
Chingis Maksimov ${ }^{\mathrm{a}}$, Alexander Melnikov ${ }^{\mathrm{b}}$ \\ ${ }^{a}$ Canadian Western Bank, Edmonton, AB, Canada \\ bUniversity of Alberta, Edmonton, $A B$, Canada \\ bhttps://orcid.org/0000-0001-6261-0806 \\ ${ }^{b}$ Web of Science ResearcherID P-4620-2017
}

\begin{abstract}
It is widely accepted to use conditional value-at-risk for risk management needs and option pricing. As a rule, there are difficulties in exact calculations of conditional value-at-risk. In the paper, we use the conditional value-at-risk methodology to price spread options, extending some approximation approaches for these needs. Our results we illustrate by numerical calculations which demonstrate their effectiveness. We also show how conditional value-at-risk pricing can help with regulatory needs inspired by the Basel Accords.

Keywords: spread options; conditional value-at-risk; approximation methods; capital constraints; Margrabe market
\end{abstract}

For citation: Maksimov, C., \& Melnikov, A. (2021). On approximate pricing of spread options via conditional value-at-risk. Review of Business and Economics Studies, 9(3),27-51. DOI: 10.26794/2308-944X-2021-9-3-27-51

\section{Приближенное оценивание опционов обмена акций через ожидаемые потери}

\author{
Чингис Максимов ${ }^{\mathrm{a}}$, Александр Мельников ${ }^{\mathrm{b}}$ \\ aCanadian Western Bank, Эдмонтон, АВ, Канада \\ ьУниверситет Альберты, Эдмонтон, АВ, Канада
}

\begin{abstract}
АННОТАЦИЯ
Как правило, существуют сложности для точного определения уровня ожидаемых потерь в целях управления риском и для оценивания опционов. В данной работе использована методология ожидаемых потерь для оценки опционов обмена акций с помощью некоторых приближений. Эффективность результатов наглядно продемонстрирована численными расчетами. Показано, как анализ ожидаемых потерь может помочь в выполнении требований постановлений Базельских соглашений III.

Ключевые слова: варианты распространения; условная стоимость, подверженная риску; методы аппроксимации; нехватка капитала; Рынок Марграбе
\end{abstract}

(c) Chingis Maksimov, Alexander Melnikov, 2021 


\section{Introduction}

In complete markets, every contingent claim is replicable in the class of self-financing strategies, and its price is unique. However, there is a whole range of arbitrage-free prices in incomplete markets or in markets with constraints. The minimum price that guarantees no underhedging at maturity is equal to the supremum of this price range. The resulting strategy is known as superhedging (see, for instance, El Karoui and Quenez (1995)). An investor can choose to stay within the boundaries of perfect hedging and completely eliminate potential risks by engaging in superhedging strategies. But the cost of such a strategy can be too high to be implemented successfully. A viable alternative is to accept the possibility of a shortfall - the difference between the payoff of the contingent claim and the replicating portfolio at maturity. This approach is usually exploited when there are market constraints on the amount of capital that can be used for hedging. It has practical benefits as regulators frequently require financial institutions to use a certain amount of funds conservatively to be able to meet their obligations. Still, the extra funds saved on hedging can be used more aggressively in an attempt to earn an extra return. Two main approaches have been considered in the literature. The first one includes maximising the probability of a successful hedge. One of the earliest works is by Kulldorf (1993). The author considered a stochastic control problem with a single risky asset whereby an agent aims to reach a particular value of fortune on a finite time interval before first going broke. Browne (1999) expanded upon the results obtained by Kulldorf (1993). The author considered a market setting with several risky securities and determined the optimal policy that maximises the probability of reaching a certain level of wealth before some fixed terminal time. Working in this direction, Foellmer and Leukert (1999) transformed the initial problem into the problem when an optimal strategy maximises the probability of successful hedging. The resulting strategy can be viewed as a dynamic version of the Value-at-Risk (VaR) concept, a popular measure of market risk exposure. The major drawback of the approach is that the size of the potential shortfall is not taken into account. Developing the approach, Foellmer and Leukert (2000) pro- pose to minimise the amount of expected shortfall where some loss function $l$ measures an investor's attitude to the size of the shortfall. The key idea is to use the Neyman-Pearson lemma to modify the original contingent claim so that the modified contingent claim can be perfectly hedged. The authors show that the modified claim's perfect hedging strategy is also the optimal strategy for the initial minimisation problem.

The methodology proposed by Foellmer and Leukert (2000) leaves some space for a choice of the loss function to model the attitude of the investor towards the potential shortfall. Value-atrisk (VaR), being the most popular tool for measuring market risk exposure by practitioners, is a natural choice. However, the use of VaR was severely criticised for failing to predict the scope of the losses during the global financial crisis. The most recent Basel III framework has signified the major shift from VaR to conditional Value-at-Risk (CVaR) as the encouraging measure of risk under stress. According to the Basel Committee on Banking Supervision (2016), the use of CVaR "will help to ensure a more prudent capture of 'tail risk' and capital adequacy during periods of significant financial market stress." CVaR has some beneficial mathematical properties that VaR lacks. First of all, CVaR satisfies the four properties of translation invariance, subadditivity, positive homogeneity, and monotonicity, making it a coherent measure of risk (Artzner et al., 1999)). In general, VaR does not satisfy the subadditivity property unless the joint distribution function of portfolio losses is from a family of elliptical distributions. Another advantage of CVaR over VaR is that it is a spectral measure of risk (Acerbi, 2002)), meaning that it directly relates to the notion of risk-aversion, an essential concept in studying optimal consumption problems through the use of utility functions. One major drawback of CVaR is that it, in its original form, is a hard risk measure to optimise with respect to. According to Brutti Righi and Ceretta (2016), "despite the advantages of ES, this measure is less frequently utilised than VaR because forecasting ES is challenging due to its complex definition", where ES stands for the same concept as CVaR. However, Rockafellar and Uryasev (2000) showed that an intrinsic relation between the two risk measures exists and developed a methodology for optimising an investment portfolio with 
respect to both VaR and CVaR simultaneously. The central idea is to introduce an auxiliary function $F$ through which VaR and CVaR can be expressed. The properties of convexity and continuous differentiability make the function $F$ "well-behaved" for optimisation tasks. Melnikov and Smirnov (2012) applied the ideas of Foellmer and Leukert (2000) to the case where CVaR represents the loss function $l$ that models the attitude of an agent to risk and considered the following dual problem: minimisation of CVaR when the initial capital is bounded from above, and minimisation of hedging costs subject to a constraint of the amount of CVaR. The authors further used the representation of CVaR as in Rockafellar and Uryasev (2000). The explicit results were obtained within the framework of the Black-Scholes market with a single risky asset.

This paper aims to take a step in the direction of generalising the results obtained by Melnikov and Smirnov (2012) and consider the problem of CVaR-based option pricing within the context of the Margrabe market model with two risky assets. The option type of interest is a plain vanilla spread option. Spread options are broadly used and appear in a wide range of financial markets: as crack spread option in energy markets, as credit spread options in fixed income markets, and as options to exchange one asset for another in equity markets (see Margrabe, 1978; Fischer, 1978). The problem is further complicated in several aspects. For example, a non-trivial aspect of pricing such options requires knowing the probability distribution of the difference between log-normal random variables that do not admit a satisfactory theoretical expression. Hence, some approximation methods are necessary. In particular, the paper utilises the approximate spread option pricing methodology proposed by Bjerksund and Stensland (2006) and an approximation based on the assumption that the difference between two log-normal random variables is normally distributed. Furthermore, CVaR is chosen as the measure of risk to make the paper's results easily applicable by practitioners in the industry.

\section{Preliminaries and Existing Approximating Methods}

Let $\left(\Omega, \mathcal{F}, F=(\mathcal{F}(t))_{t \geq 0}, P\right)$ be a standard stochastic basis with filtration $\mathcal{F}(t)$ that satisfies the usual conditions, and $\mathcal{F}(0)=\{\Omega, \varnothing\}$. Assume that $T$ is the terminal time for all the contingent claims traded on this market. Then the dynamics of the two stock price processes $S_{1}=\left(S_{1}(t): t \in[0, T]\right)$ and $S_{2}=\left(S_{2}(t): t \in[0, T]\right)$ are assumed to satisfy the following stochastic differential equations (SDEs):

$$
\begin{aligned}
& d S_{1}(t)=S_{1}(t)\left[\mu_{1} d t+\sigma_{1} d W_{1}(t)\right] \\
& d S_{2}(t)=S_{2}(t)\left[\mu_{2} d t+\sigma_{2} d W_{2}(t)\right]
\end{aligned}
$$

where $W_{1}=\left(W_{1}(t): t \in[0, T]\right)$ and $W_{2}=\left(W_{2}(t): t \in[0, T]\right)$ are standard Brownian motion processes with correlation coefficient $\rho$.

The original Margrabe market model only assumed the existence of two risky assets and no bank account. Therefore, we take all the stocks traded in this market as already discounted.

We further assume that the market is arbitrage-free and complete and introduce a unique equivalent martingale measure $Q$ via the Radon-Nikodym derivative:

$$
Z(T)=\frac{d Q}{d P}
$$

We say that measure $Q$ is equivalent to measure $P$ if the two measures agree on the sets of measure 0 , i.e., if

$$
P(w)>0 \Leftrightarrow Q(w)>0 .
$$

The process $Z=(Z(t): t \in[0, T])$ takes the following functional form (see, for instance, Melnikov (2011)):

$$
Z(t)=\exp \left[\phi_{1} W_{1}(t)+\phi_{2} W_{2}(t)-\frac{\sigma_{\phi}^{2}}{2} t\right],
$$


where

$$
\begin{array}{r}
\phi_{1}=\frac{\sigma_{1} \mu_{2} \rho-\sigma_{2} \mu_{1}}{\sigma_{1} \sigma_{2}\left(1-\rho^{2}\right)}, \\
\phi_{2}=\frac{\sigma_{2} \mu_{1} \rho-\sigma_{1} \mu_{2}}{\sigma_{1} \sigma_{2}\left(1-\rho^{2}\right)}, \\
\sigma_{\phi}^{2}=\phi_{1}^{2}+\phi_{2}^{2}+2 \rho \phi_{1} \phi_{2} .
\end{array}
$$

Under the risk-neutral probability measure $Q$, the dynamics of the two assets satisfy

$$
\begin{aligned}
& d S_{1}(t)=S_{1}(t) \sigma_{1} d W_{1}^{Q}(t), \\
& d S_{2}(t)=S_{2}(t) \sigma_{2} d W_{2}^{Q}(t),
\end{aligned}
$$

where $W_{1}^{Q}=\left(W_{1}^{Q}(t): t \in[0, T]\right)$ and $W_{2}^{Q}=\left(W_{2}^{Q}(t): t \in[0, T]\right)$ are, according to the Girsanov theorem (Shreve (2011)), standard Brownian motion processes with correlation coefficient $\rho$. We can rewrite the process $Z$ under the measure $Q$ as follows:

$$
Z(t)=\exp \left[\phi_{1} W_{1}^{Q}(t)+\phi_{2} W_{2}^{Q}(t)-\left(\frac{\sigma_{\phi}^{2}}{2}+\phi_{1} \theta_{1}+\phi_{2} \theta_{2}\right) t\right],
$$

where

$$
\begin{gathered}
\theta_{1}=\frac{\mu_{1}}{\sigma_{1}}, \\
\theta_{2}=\frac{\mu_{2}}{\sigma_{2}} .
\end{gathered}
$$

The general payoff function of a spread option is of the following form:

$$
\left[S_{1}(T)-S_{2}(T)-K\right]^{+}
$$

where $K$ is a deterministic strike price. The exact pricing formula for the special case when $K=0$ was determined independently by Margrabe (1978) and Fischer (1978). The price of such a contingent claim is given by

$$
p=S_{1}(0) \Phi\left(d_{1}\right)-S_{2}(0) \Phi\left(d_{2}\right)
$$

where

$$
\begin{array}{r}
d_{1}=\frac{\ln \left(\frac{S_{1}(0)}{S_{2}(0)}\right)+\frac{\sigma^{2} T}{2}}{\sigma \sqrt{T}}, \\
d_{2}=d_{1}-\sigma \sqrt{T}, \\
\sigma=\sqrt{\sigma_{1}^{2}+\sigma_{2}^{2}-2 \sigma_{1} \sigma_{2} \rho},
\end{array}
$$

and $\Phi(x)$ is the standard normal cumulative distribution function (CDF). To avoid ambiguity, we will refer to the special case of equation (5) as an option to exchange one asset for another one; and as a spread option otherwise.

However, it is generally accepted that the probability density function (PDF) of linear combinations of log-normal random variables does not have a closed form. Approximations of the distribution of sums of log-normal random variables exist in the literature; see, for example, Mehta et al. (2007), Cobb and Rumi (2012), Hcine and Bouallegue (2015). Less is known about the distribution of the difference between cor- 
related log-normal random variables. Lo (2012) proposed the Lie-Trotter operator splitting method and found that a shifted log-normal process governs the difference between two log-normal random variables. A more recent work by Gulisashvili and Tankov (2016) considers the tail behaviour of the distributions of linear combinations of log-normal random variables explicitly. The results of the paper allow approximating the probabilities of tail events directly. The authors further provide insights into how these findings can be applied in the domain of risk management.

Thus, only approximate pricing formulas for equation (5) exist. Carmona and Durrleman (2003) provide a thorough overview of spread option pricing methodologies. However, while most of the approximations to equation (5) that exist in the literature provide accurate estimates, these are not always easily transferable to the domain of risk management due to their complexity. For the purposes of this paper, we will work around the idea of approximating the difference between two log-normal random variables using a normal distribution. According to Carmona and Durrleman (2003), "computing histograms of historical spread values shows that the marginal distribution of a spread at a given time extends on both tails, and surprisingly enough, that the normal distribution can give a reasonable fit." It allows us to price an option with a payoff as in equation (5) in the approximate form, similar to equation (6). Consider the difference between the two stock prices at maturity:

$$
S_{1}(T)-S_{2}(T)=S_{1}(0) \exp \left[-\frac{\sigma_{1}^{2} T}{2}+\sigma_{1} W_{1}^{Q}(T)\right]-S_{2}(0) \exp \left[-\frac{\sigma_{2}^{2} T}{2}+\sigma_{2} W_{2}^{Q}(T)\right] .
$$

The above expression represents the difference between two log-normal random variables distribution of which is not log-normal and generally has not been determined. By applying Taylor series expansion to the exponents,

$$
S_{1}(T)-S_{2}(T)=S_{1}(0)-S_{2}(0)+S_{1}(0) z_{1}-S_{2}(0) z_{2}+S_{1}(0) \sum_{n=2}^{\infty} \frac{z_{1}^{n}}{n !}-S_{2}(0) \sum_{n=2}^{\infty} \frac{z_{2}^{n}}{n !},
$$

where

$$
\begin{aligned}
& z_{1}=-\frac{\sigma_{1}^{2} T}{2}+\sigma_{1} W_{1}^{Q}(T) \sim N\left(-\frac{\sigma_{1}^{2} T}{2}, \sigma_{1}^{2} T\right), \\
& z_{2}=-\frac{\sigma_{2}^{2} T}{2}+\sigma_{2} W_{2}^{Q}(T) \sim N\left(-\frac{\sigma_{2}^{2} T}{2}, \sigma_{2}^{2} T\right) .
\end{aligned}
$$

Equation (8) represents a normal random variable plus an error term in the amount of $S_{1}(0) \sum_{n=2}^{\infty} \frac{z_{1}^{n}}{n !}-S_{2}(0) \sum_{n=2}^{\infty} \frac{z_{2}^{n}}{n !}$. The price of the option with a payoff as in equation (5) can then be approximated as follows:

$$
p=S_{1}(0) \Phi\left(d_{1}\right)-S_{2}(0) \Phi\left(d_{2}\right)-K \Phi\left(d_{3}\right),
$$

where

$$
\begin{aligned}
& d_{1} \quad=\frac{-K-m}{\sigma}+\sigma_{1} \rho_{1} \sqrt{T}, \\
& d_{2}=\frac{-K-m}{\sigma}+\sigma_{2} \rho_{2} \sqrt{T}, \\
& =\frac{-K-m}{\sigma}, \\
& d_{3} \quad \\
& \rho_{1}=\frac{\left[S_{1}(0) \sigma_{1}-S_{2}(0) \sigma_{2} \rho\right] \sqrt{T}}{\sigma}, \\
& \rho_{2}=\frac{\left[S_{1}(0) \sigma_{1} \rho-S_{2}(0) \sigma_{2}\right] \sqrt{T}}{\sigma},
\end{aligned}
$$


and where $S_{1}(T)-S_{2}(T) \approx \gamma \sim N\left(m, \sigma^{2}\right)$, i.e., where the difference $S_{1}(T)-S_{2}(T)$ in the indicator function of the option, exercise event is replaced by a normal random variable $\gamma$ with mean $m$ and variance $\sigma^{2}$. We can use the moment matching technique to calculate the moments of $\gamma$. Consider the mean $m$,

$$
\begin{aligned}
& m=E_{Q}\left[S_{1}(T)-S_{2}(T)\right]= \\
& =E_{Q}\left[S_{1}(0) \exp \left(-\frac{\sigma_{1}^{2} T}{2}+\sigma_{1} W_{1}^{Q}(T)\right)\right]-E_{Q}\left[S_{2}(0) \exp \left(-\frac{\sigma_{2}^{2} T}{2}+\sigma_{2} W_{2}^{Q}(T)\right)\right] \\
& =S_{1}(0)-S_{2}(0) .
\end{aligned}
$$

The corresponding variance is

$$
\begin{array}{r}
\sigma^{2}=\operatorname{Var}\left[S_{1}(T)-S_{2}(T)\right]=S_{1}^{2}(0) \exp \left(\sigma_{1}^{2} T\right)+S_{2}^{2}(0) \exp \left(\sigma_{2}^{2} T\right)- \\
2 S_{1}(0) S_{2}(0) \exp \left[-\left(\frac{\sigma_{1}^{2}+\sigma_{2}^{2}}{2}\right) T+\frac{\sigma_{1}^{2} T+\sigma_{2}^{2} T+2 \sigma_{1}^{2} \sigma_{2}^{2} \rho T}{2}\right]-\left[S_{1}(0)-S_{2}(0)\right]^{2} .
\end{array}
$$

Let us call the approximation in equation (9) as a normal approximation.

The second spread option pricing formula that we are considering in the paper was proposed by Bjerksund and Stensland (2006), where the authors consider the following expectation:

$$
E_{Q}\left[\left(S_{1}(T)-S_{2}(T)-K\right) I_{\left\{S_{1}(T) \geq \frac{c\left(S_{2}(T)\right)^{2}}{E_{Q}\left[\left(S_{2}(T)\right)^{2}\right]}\right\}}\right],
$$

where

$$
\begin{aligned}
& c=S_{2}(0)+K, \\
& b=\frac{S_{2}(0)}{S_{2}(0)+K} .
\end{aligned}
$$

The strategy to exercise the option depends on the price of the long asset at maturity exceeding the power function of the short asset times a constant term. The price of the spread option is then given by

$$
p=S_{1}(0) \Phi\left(d_{1}\right)-S_{2}(0) \Phi\left(d_{2}\right)-K \Phi\left(d_{3}\right)
$$

where

$$
\begin{gathered}
d_{1}=\frac{\ln \left(\frac{S_{1}(0)}{c}\right)+\left(\frac{\sigma_{1}^{2}}{2}-\sigma_{1} \sigma_{2} b \rho+\frac{\sigma_{2}^{2} b^{2}}{2}\right) T}{\sigma \sqrt{T}}, \\
d_{2}=\frac{\ln \left(\frac{S_{1}(0)}{c}\right)+\left(\frac{\sigma_{1}^{2}}{2}+\sigma_{1} \sigma_{2} \rho+\frac{\sigma_{2}^{2} b^{2}}{2}-\sigma_{2}^{2} b\right) T}{\sigma \sqrt{T}}, \\
d_{3}=\frac{\ln \left(\frac{S_{1}(0)}{c}\right)+\left(-\frac{\sigma_{1}^{2}}{2}+\frac{\sigma_{2}^{2} b^{2}}{2}\right) T}{\sigma \sqrt{T}} \\
\sigma=\sqrt{\sigma_{1}^{2}-2 \sigma_{1} \sigma_{2} b \rho+\sigma_{2}^{2} b^{2}}
\end{gathered}
$$


The authors showed, via numerical simulations, that equation (11) provides a very accurate lower bound to the true price of the contingent claim. It offers better estimates than the widely used Kirk's approximation (1995). Let us call the approximation as the BS-approximation. The derivation of equation (11) is in Appendix C.

To compare the two pricing formulas, we have estimated the prices by first varying the volatility of the first stock $\sigma_{1}$ and the time to maturity $T$ parameters. The other parameters used are as follows: $S_{1}(0)=105, S_{2}(0)=100, K=5, \sigma_{2}=0.2, \rho=0.5$. The results we present in Table 2 (refer to Appendix B). Tables 3 and 4 show the absolute and percentage errors' values compared to Monte Carlo simulations. We can infer from the tables that the percentage errors vary significantly depending on the choice of market parameters for the proposed normal approximation, whereas the BS-approximation provides more accurate estimates. Lower rates of error are associated with a shorter time to maturity and the volatility parameters of the two stocks being closer to each other. Both pricing methodologies provide the lower bound on the option price compared to Monte Carlo simulations.

CVaR-hedging Methodology Adapted to Model (1)

Consider an $\mathcal{F}(T)$-measurable European style contingent claim $H \in L^{1}(Q)$, i.e. $E_{Q}(|H|)<\infty$, with the following payoff structure:

$$
H=\left[S_{1}(T)-S_{2}(T)\right]^{+}
$$

Suppose that a financial institution has sold this option in the market and received $H(0)=E_{Q}(H)$, the amount required for perfect hedging, given by equation (6). However, the institution decides not to use all the proceeds from the sale of the option and thus is faced with the possibility of a shortfall at maturity. The question arises: What is the best trading strategy the institution should follow to minimise its expected shortfall if it uses CVaR as a measure of risk?

Denote by $\mathcal{A}$ the class of admissible self-financing trading strategies $\pi=(V(0), \xi, \eta)$, where $V(0)>0$ is the amount of initial capital, $\xi$ and $\eta$ denote the number of units of the first and second stocks held in portfolio, respectively, such that

$$
V(t)=V(0)+\int_{0}^{t} \xi(s) d S_{1}(s)+\int_{0}^{t} \eta(s) d S_{2}(s), \forall t \in[0, T], P-\text { a.s. }
$$

Strategy $\pi$ is admissible if $V(t) \geq 0, \forall t \in[0, T], P-$ a.s.

Denote by $\widehat{V}(0)$ the amount available for hedging such that $\widehat{V}(0)<H(0)$. Then the amount of shortfall $L(\pi)$ associated with a given portfolio $\pi$ takes the following form:

$$
L(\pi)=H-V(T)=H-V(0)-\int_{0}^{T} \xi(s) d S_{1}(s)-\int_{0}^{T} \eta(s) d S_{2}(s) .
$$

Fix a confidence level $\mathcal{L}$, usually $90 \%, 95 \%$ or $99 \%$ for practical purposes. We will be minimising $C V a R_{\mathcal{L}}$ over all strategies $\pi \in \mathcal{A}$ with the restriction on the amount of capital available, $V(0) \leq \dot{V}(0)$, i.e.

$$
\left\{\begin{array}{l}
\operatorname{CVaR}_{\mathcal{L}}(\pi) \rightarrow \min _{\pi} \\
\pi \in \mathcal{A}, V(0) \leq \widehat{V}(0)
\end{array}\right.
$$

Denote by $\mathcal{A}(\widehat{V}(0))$ the set of all admissible self-financing strategies that use no more initial capital than $\widehat{V}(0)$. Let us introduce an auxiliary function $F$ as follows:

$$
F_{\mathcal{L}}(\pi, z)=z+\frac{1}{1-\mathcal{L}} E\left[(L(\pi)-z)^{+}\right] .
$$


According to Rockafellar and Urysev (2000), $\mathrm{CVaR}_{\mathcal{L}}(\pi)$ and $F_{\mathcal{L}}(\pi, z)$ are interconnected through the following nice property: function $F_{\mathcal{L}}(\pi, z)$ is finite and convex with respect to $z \in \mathbb{R}$, and

$$
\operatorname{CVaR}_{\mathcal{L}}(\pi)=\min _{z \in \mathbb{R}} F_{\mathcal{L}}(\pi, z) .
$$

Moreover, minimising $\operatorname{CVaR}_{\mathcal{L}}(\pi)$ over all strategies $\pi \in \mathcal{A}(\widehat{V}(0))$ is equivalent to minimizing $F_{\mathcal{L}}(\pi, z)$ over all $(\pi, z) \in \mathcal{A}(\widehat{V}(0)) \times \mathbb{R}$ :

$$
\min _{\pi \in \mathcal{A}(V(0))} \operatorname{CVaR}_{\mathcal{L}}(\pi)=\min _{(\pi, z) \in \mathcal{A}(V(0)) \times \mathbb{R}} F_{\mathcal{L}}(\pi, z) .
$$

We arrive at the following equality:

$$
\min _{\pi \in \mathcal{A}(\hat{V}(0))} C V a R_{\mathcal{L}}(\pi)=\min _{z \in \mathbb{R}}\left\{\min _{\pi \in \mathcal{A}(V(0))}\left[z+\frac{1}{1-\mathcal{L}} E(H-V(T)-z)^{+}\right]\right\} .
$$

The expression in equation (18) represents a new optimisation objective. Let us define an auxiliary function $c(z)$ in the following way:

$$
\left.c(z)=\min _{\pi \in \mathcal{A}(\hat{V}(0))}\left[z+\frac{1}{1-\mathcal{L}} E(H-V(T)-z)^{+}\right],\right]
$$

and rewrite equation (18) in terms of the new function $c(z)$ as follows:

$$
\min _{\pi \in \mathcal{A}(\hat{V}(0))} \operatorname{CVaR}_{\mathcal{L}}(\pi)=\min _{z \in \mathbb{R}} c(z) .
$$

Let the minimum value of the function $c(z)$ for each $z$ be achieved using strategy

$$
\tilde{\pi}(z)=(\tilde{V}(0, z), \tilde{\xi}(z), \tilde{\eta}(z))
$$

We then have:

$$
\min _{\pi \in \mathcal{A}(\hat{V}(0))} E(H-V(T)-z)^{+}=E(H-\tilde{V}(T, z)-z)^{+},
$$

where

$$
\tilde{V}(T, z)=\tilde{V}(0, z)+\int_{0}^{T} \tilde{\xi}(s, z) d S_{1}(s)+\int_{0}^{T} \tilde{\eta}(s, z) d S_{2}(s)
$$

Suppose that the global minimum of the function $c(z)$ is achieved at the point $\tilde{z}$, i.e.,

$$
\min _{z \in \mathbb{R}} c(z)=c(\tilde{z}) \text {. }
$$

Then the optimal solution to the problem of $C V a R_{\mathcal{L}}$ minimization over all $\pi \in \mathcal{A}(\widehat{V}(0))$, set in equation (15), is the strategy

$$
\tilde{\pi}(\tilde{z})=\{\tilde{V}(0, \tilde{z}), \tilde{\xi}(\tilde{z}), \tilde{\eta}(\tilde{z})\}
$$

Now, according to equation (17), we have:

$$
\operatorname{CVaR}_{\mathcal{L}}(\tilde{\pi})=c(\tilde{z})
$$

It follows that if we can find the strategy $\tilde{\pi}$ in an explicit form, then the problem of $C V a R_{\mathcal{L}}$ minimisation will be reduced to the problem of minimisation of the function $c(z)$. Observe that for each $z$, the strategy $\tilde{\pi}$ is a solution to the following problem 


$$
E(H-V(T)-z)^{+} \rightarrow \min _{\pi \in \mathcal{A}(\hat{V}(0))}
$$

Let us note that

$$
(H-V(T)-z)^{+}=\left[(H-z)^{+}-V(T)\right]^{+} .
$$

Denote $(H-z)^{+}$by $H(z)$. It is evident that $H(z)$ is an $\mathcal{F}$-measurable random variable, $H(z) \in L^{1}(Q)$ and $H(z) \geq 0$. We can consider $H(z)$ as a contingent claim. Equation (22) can be reformulated in the following form:

$$
E(H(z)-V(T))^{+} \rightarrow \min _{\pi \in \mathcal{A}(\widehat{V}(0))}
$$

This optimisation problem can be interpreted as the problem of expected shortfall minimisation over the strategy set $\mathcal{A}(\widehat{V}(0))$ of contingent claim $H(z)$, which was solved by Melnikov and Smirnov (2012). The optimal solution $\tilde{\pi}=(\tilde{V}(0), \tilde{\xi}, \tilde{\eta})$ of the problem of expected shortfall minimisation is the perfect hedge of the modified contingent claim $\tilde{H}(z)=\tilde{\varphi}(z)(H-z)^{+}$or, equivalently, $\tilde{H}(z)=\tilde{\varphi}(z) H(z)$ :

$$
E_{Q}(\tilde{H}(z) \mid \mathcal{F}(\mathrm{t}))=\tilde{V}(0, z)+\int_{0}^{T} \tilde{\xi}(s, z) d S_{1}(s)+\int_{0}^{T} \tilde{\eta}(s, z) d S_{2}(s), \forall t \in[0, T], P-\text { a.s. },
$$

where

$$
\begin{array}{r}
\tilde{\varphi}(z)=I_{\left\{\frac{d P}{d Q}>\tilde{a}(z)\right\}}+\beta(z) I_{\left\{\frac{d P}{d Q}=\tilde{a}(z)\right\}}, \\
\tilde{a}(z)=\inf \left\{a \geq 0: E_{Q}\left[(H-z)^{+} I_{\left\{\frac{d P}{d Q}>a\right\}}\right] \leq \widehat{V}(0)\right\}, \\
\beta(z)=\frac{\widehat{V}(0)-E_{Q}\left[(H-z)^{+} I_{\left\{\frac{d P}{d Q}>\tilde{a}(z)\right\}}\right]}{E_{Q}\left[(H-z)^{+} I_{\left.\left\{\frac{d P}{d Q}=\tilde{a}(z)\right\}\right]}\right]} .
\end{array}
$$

Moreover, in the context of equation (21), the function $c(z)$ admits the following description:

$$
c(z)=\left\{\begin{array}{c}
z+\frac{1}{1-\mathcal{L}} E\left[(1-\tilde{\varphi}(z))(H-z)^{+}\right], z<\hat{z} \\
z, z \geq \hat{z}
\end{array}\right.
$$

Equivalently,

$$
c(z)=\left\{\begin{array}{c}
z+\frac{1}{1-\mathcal{L}} E[H(z)-\tilde{H}(z)], z<\hat{z} \\
z, z \geq \hat{z}
\end{array}\right.
$$

where $\hat{z}$ is the solution to the following equation:

$$
\widehat{V}(0)=E_{Q}\left[(H-z)^{+}\right] .
$$

\section{Main Results: Extended Approximate Formulas}

To find the price of the optimal hedge, in CVaR sense, or, equivalently, to construct a replicating portfolio with the lowest level of $C V a R_{\mathcal{L}}$, we must first find $\hat{z}$ in equation (26). To do it, we will be using the proposed normal approximation, and the BS-approximation described above. Once we have determined 
the unique value $\hat{z}$, we can minimise equation (25) numerically, using the Monte Carlo simulation technique. Suppose that $\tilde{z}$ is the global minimum of the function $c(z)$. Noting that the distribution of Brownian motion is atomless, the problem is reduced to evaluating the following expectation:

$$
E_{Q}(\tilde{H}(\tilde{z}))=E_{Q}\left[\left(S_{1}(T)-S_{2}(T)-\tilde{z}\right)^{+} I_{\left\{\frac{d P}{d Q}>\tilde{a}(\tilde{z})\right\}}\right] .
$$

Depending on the chosen approximating method, the following two theorems provide the necessary tools for constructing a hedge with the lowest level of $C V a R_{\mathcal{L}}$ :

Theorem 1. Approximating the distribution of the difference between two log-normally distributed stock prices as a normal distribution, the price $p$ of setting up a replicating portfolio for a spread option at any time $t \leq T$ can be estimated as follows:

$$
p=S_{1}(0) \Phi^{2}\left(\hat{x}_{1}, \hat{y}_{1}, \rho_{3}\right)-S_{2}(0) \Phi^{2}\left(\hat{x}_{2}, \hat{y}_{2}, \rho_{3}\right)-\tilde{z} \Phi^{2}\left(\hat{x}_{3}, \hat{y}_{3}, \rho_{3}\right)
$$

where

$$
\begin{aligned}
& \hat{x}_{1}=\frac{m-\tilde{z}}{\sigma}+\sigma_{1} \rho_{1} \sqrt{T}, \\
& \hat{y}_{1}=\tilde{K}+\sigma_{1} \rho_{4} \sqrt{T} \text {, } \\
& \hat{x}_{2}=\frac{m-\tilde{z}}{\sigma}+\sigma_{2} \rho_{2} \sqrt{T} \text {, } \\
& \hat{y}_{2}=\tilde{K}+\sigma_{2} \rho_{5} \sqrt{T}, \\
& \hat{x}_{3}=\frac{m-\tilde{z}}{\sigma}, \\
& \hat{y}_{3}=\tilde{K}, \\
& \rho_{1}=\frac{\sqrt{T}}{\sigma}\left(S_{1}(0) \sigma_{1}-S_{2}(0) \sigma_{2} \rho\right) \text {, } \\
& \rho_{2}=\frac{\sqrt{T}}{\sigma}\left(S_{1}(0) \sigma_{1} \rho-S_{2}(0) \sigma_{2}\right) \text {, } \\
& \rho_{3}=\frac{a-b}{\sigma}, \\
& \rho_{4}=-\frac{\rho \phi_{2}+\phi_{1}}{\sigma_{\phi}}, \\
& \rho_{5}=-\frac{\phi_{2}+\phi_{1} \rho}{\sigma_{\phi}}, \\
& \sigma_{\phi}=\sqrt{\phi_{1}^{2}+\phi_{2}^{2}+2 \rho \phi_{1} \phi_{2}}, \\
& a=S_{2}(0) \frac{\sigma_{2} \sqrt{T}}{\sigma_{\phi}}\left(\phi_{1} \rho+\phi_{2}\right) \text {, } \\
& b=S_{1}(0) \frac{\sigma_{1} \sqrt{T}}{\sigma_{\phi}}\left(\phi_{1}+\phi_{2} \rho\right), \\
& \tilde{K}=\frac{\left(\frac{\sigma_{\phi}^{2}}{2}+\phi_{1} \theta_{1}+\phi_{2} \theta_{2}\right) T+\ln \left(\frac{1}{\tilde{a}(\tilde{z})}\right)}{\sigma_{\phi} \sqrt{T}}, \\
& S_{1}(T)-S_{2}(T) \approx \gamma \sim N\left(m, \sigma^{2}\right),
\end{aligned}
$$


and $\Phi^{2}\left(\mathrm{x}_{1}, \mathrm{x}_{2}, \rho\right)$ is the CDF of the bivariate standard normal distribution with correlation $\rho$.

Proof. We want to find the price of the option with the following payoff

$$
\left(S_{1}(T)-S_{2}(T)-K\right)^{+} I_{\left\{\frac{d P}{d Q}>\tilde{a}(\tilde{z})\right\}} \cdot
$$

Let us first consider the expression in the indicator function:

$$
\begin{aligned}
\left\{\frac{d P}{d Q}>\tilde{a}(\tilde{z})\right\} & =\left\{\frac{1}{\tilde{a}(\tilde{z})}>\exp \left[\phi_{1} W_{1}^{Q}(T)+\phi_{2} W_{2}^{Q}(T)-\left(\frac{\sigma_{\phi}^{2}}{2}+\phi_{1} \theta_{1}+\phi_{2} \theta_{2}\right) T\right]\right\} \\
& =\left\{\frac{\phi_{1} W_{1}^{Q}(T)+\phi_{2} W_{2}^{Q}(T)}{\sigma_{\phi} \sqrt{T}}<\frac{\left(\frac{\sigma_{\phi}^{2}}{2}+\phi_{1} \theta_{1}+\phi_{2} \theta_{2}\right) T+\ln \left(\frac{1}{\tilde{a}(\tilde{z})}\right)}{\sigma_{\phi} \sqrt{T}}\right\} \\
& =\{\in<\tilde{K}\},
\end{aligned}
$$

where

$$
\begin{gathered}
\in=\frac{\tilde{W}^{Q}(T)}{\sqrt{T}} \sim N(0,1), \\
\tilde{W}^{Q}(T)=\frac{\phi_{1} W_{1}^{Q}(T)+\phi_{2} W_{2}^{Q}(T)}{\sigma_{\phi}}, \\
\tilde{K}=\frac{\left(\frac{\sigma_{\phi}^{2}}{2}+\phi_{1} \theta_{1}+\phi_{2} \theta_{2}\right) T+\ln \left(\frac{1}{\tilde{a}(\tilde{z})}\right)}{\sigma_{\phi} \sqrt{T}}, \\
\phi_{1}=\frac{\sigma_{1} \mu_{2} \rho-\sigma_{2} \mu_{1}}{\sigma_{1} \sigma_{2}\left(1-\rho^{2}\right)}, \\
\phi_{2}=\frac{\sigma_{2} \mu_{1} \rho-\sigma_{1} \mu_{2}}{\sigma_{1} \sigma_{2}\left(1-\rho^{2}\right)}, \\
\sigma_{\phi}=\sqrt{\phi_{1}^{2}+\phi_{2}^{2}+2 \phi_{1} \phi_{2} \rho}, \\
\theta_{1}=\frac{\mu_{1}}{\sigma_{1}}, \\
\theta_{2}=\frac{\mu_{2}}{\sigma_{2}} .
\end{gathered}
$$

Replacing $S_{1}(T)-S_{2}(T)$ by $\gamma \sim N\left(m, \sigma^{2}\right)$, we obtain: 


$$
\begin{gathered}
E_{Q}\left[\left(S_{1}(T)-S_{2}(T)-K\right)^{+} I_{\left\{\frac{d P}{d Q}>\tilde{a}(\tilde{z})\right\}}\right] \\
=E_{Q}\left[\left(S_{1}(T)-S_{2}(T)-K\right) I_{\left\{S_{1}(T)-S_{2}(T)>K\right\}} I_{\left\{\frac{d P}{d Q}>\tilde{a}(\tilde{z})\right\}}\right] \\
=E_{Q}\left[S_{1}(T) I_{\{\gamma>K\}} I_{\{\epsilon<\tilde{K}\}}\right]-E_{Q}\left[S_{2}(T) I_{\{\gamma>K\}} I_{\{\epsilon<\tilde{K}\}}\right]-K E_{Q}\left[I_{\{\gamma>K\}} I_{\{\epsilon<\tilde{K}\}}\right] \\
=S_{1}(0) \exp \left(-\frac{\sigma_{1}^{2} T}{2}\right) E_{Q}\left[\exp \left(\sigma_{1} W_{1}^{Q}(T)\right) I_{\{-\gamma<-K\}} I_{\{\epsilon<\tilde{K}\}}\right] \\
-S_{2}(0) \exp \left(-\frac{\sigma_{2}^{2} T}{2}\right) E_{Q}\left[\exp \left(\sigma_{2} W_{2}^{Q}(T)\right) I_{\{-\gamma<-K\}} I_{\{\epsilon<\tilde{K}\}}\right]-K E_{Q}\left[I_{\{-\gamma<-K\}} I_{\{\epsilon<\tilde{K}\}]}\right] .
\end{gathered}
$$

Consider the first term in equation (30):

$$
\begin{array}{r}
S_{1}(0) \exp \left(-\frac{\sigma_{1}^{2} T}{2}\right) E_{Q}\left[\exp \left(\sigma_{1} W_{1}^{Q}(T)\right) I_{\{-\gamma<-K\}} I_{\{\dot{o}<\tilde{K}\}}\right]= \\
S_{1}(0) \exp \left(-\frac{\sigma_{1}^{2} T}{2}\right) E_{Q}\left[\exp \left(-Z_{1}\right) I_{\{X<-K\}} I_{\{Y<\tilde{K}\}}\right],
\end{array}
$$

where

$$
\begin{array}{rr}
Z_{1} & =-\sigma_{1} W_{1}^{Q}(T) \sim N\left(0, \sigma_{1}^{2} T\right), \\
X & =-\gamma \sim N\left(-m, \sigma^{2}\right), \\
Y \quad=\frac{\phi_{1} W_{1}^{Q}(T)+\phi_{2} W_{2}^{Q}(T)}{\sigma_{\phi} \sqrt{T}} \sim N(0,1) .
\end{array}
$$

To apply the two-asset lemma (see Appendix A) to the expectation term, we need to estimate the correlation coefficients $\rho_{Z_{1} X}, \rho_{Z_{1} Y}$ and $\rho_{X Y}$. Consider $\rho_{X Y}$,

$$
\rho_{X Y}=\frac{\sigma_{x y}^{2}}{\sigma_{x} \sigma_{y}} \text {. }
$$

Since $Y \sim N(0,1)$,

$$
\begin{gathered}
\sigma_{x y}^{2}=E_{Q}(X Y) \\
=E_{Q}\left[\frac{\phi_{1} W_{1}^{Q}(T)+\phi_{2} W_{2}^{Q}(T)}{\sigma_{\phi} \sqrt{T}} S_{2}(T)\right]-E_{Q}\left[\frac{\phi_{1} W_{1}^{Q}(T)+\phi_{2} W_{2}^{Q}(T)}{\sigma_{\phi} \sqrt{T}} S_{1}(T)\right] \\
=S_{2}(0) \exp \left(-\frac{\sigma_{2}^{2} T}{2}\right) \frac{1}{\sigma_{\phi} \sqrt{T}} E_{Q}\left[\phi_{1} W_{1}^{Q}(T) \exp \left(\sigma_{2} W_{2}^{Q}(T)\right)+\phi_{2} W_{2}^{Q}(T) \exp \left(\sigma_{2} W_{2}^{Q}(T)\right)\right] \\
-S_{1}(0) \exp \left(-\frac{\sigma_{1}^{2} T}{2}\right) \frac{1}{\sigma_{\phi} \sqrt{T}} E_{Q}\left[\phi_{1} W_{1}^{Q}(T) \exp \left(\sigma_{1} W_{1}^{Q}(T)\right)+\phi_{2} W_{2}^{Q}(T) \exp \left(\sigma_{1} W_{1}^{Q}(T)\right)\right]
\end{gathered}
$$


Opening the brackets and calculating the expectations, the above yields:

$$
S_{2}(0) \frac{\sigma_{2} \sqrt{T}}{\sigma_{\phi}}\left(\phi_{1} \rho+\phi_{2}\right)-S_{1}(0) \frac{\sigma_{1} \sqrt{T}}{\sigma_{\phi}}\left(\phi_{1}+\phi_{2} \rho\right) .
$$

To get the correlation, we need to divide it by $\sigma_{x} \sigma_{y}$ to get:

$$
\rho_{X Y}=\frac{a-b}{\sigma},
$$

where

$$
\begin{aligned}
a & =S_{2}(0) \frac{\sigma_{2} \sqrt{T}}{\sigma_{\phi}}\left(\phi_{1} \rho+\phi_{2}\right), \\
b & =S_{1}(0) \frac{\sigma_{1} \sqrt{T}}{\sigma_{\phi}}\left(\phi_{1}+\phi_{2} \rho\right) .
\end{aligned}
$$

Now consider $\rho_{Z_{1} X}$,

$$
\rho_{Z_{1} X}=\frac{\sigma_{z_{1} x}^{2}}{\sigma_{z_{1}} \sigma_{y}} .
$$

Since $Z_{1} \sim N\left(0, \sigma_{1}^{2} T\right)$,

$$
\begin{gathered}
\sigma_{z_{1} x}^{2}=E_{Q}\left(Z_{1} X\right) \\
=E_{Q}\left\{\sigma_{1} W_{1}^{Q}(T)\left[S_{1}(0) \exp \left(-\frac{\sigma_{1}^{2} T}{2}+\sigma_{1} W_{1}^{Q}(T)\right)-S_{2}(0) \exp \left(-\frac{\sigma_{2}^{2} T}{2}+\sigma_{2} W_{2}^{Q}(T)\right)\right]\right\} \\
=S_{1}(0) \exp \left(-\frac{\sigma_{1}^{2} T}{2}\right) E_{Q}\left[\sigma_{1} W_{1}^{Q}(T) \exp \left(\sigma_{1} W_{1}^{Q}(T)\right)\right] \\
-S_{2}(0) \exp \left(-\frac{\sigma_{2}^{2} T}{2}\right) E_{Q}\left[\sigma_{1} W_{1}^{Q}(T) \exp \left(\sigma_{2} W_{2}^{Q}(T)\right)\right] .
\end{gathered}
$$

Opening the brackets and calculating the expectations,

$$
\sigma_{z_{1} x}^{2}=S_{1}(0) \sigma_{1}^{2} T-S_{2}(0) \sigma_{1} \sigma_{2} \rho T
$$

Similarly, by dividing by $\sigma_{x} \sigma_{y}$ we get the correlation:

$$
\rho_{Z_{1} X}=\frac{\sqrt{T}}{\sigma}\left(S_{1}(0) \sigma_{1}-S_{2}(0) \sigma_{2} \rho\right)
$$

Let us now consider $\rho_{Z_{1} Y}$,

$$
\rho_{Z_{1} Y}=\frac{\sigma_{z_{1} y}^{2}}{\sigma_{z_{1}} \sigma_{y}} .
$$

Since both random variables $Z_{1}$ and $Y$ have zero expectation, 


$$
\begin{gathered}
\sigma_{z_{1} y}^{2}=E_{Q}\left(Z_{1} Y\right) \\
=E_{Q}\left[-\sigma_{1} W_{1}^{Q}(T)\left(\frac{\phi_{1} W_{1}^{Q}(T)+\phi_{2} W_{2}^{Q}(T)}{\sigma_{\phi} \sqrt{T}}\right)\right] .
\end{gathered}
$$

Simplifying, we get:

$$
\rho_{Z_{1} Y}=-\frac{\phi_{1}+\rho \phi_{2}}{\sigma_{\phi}}
$$

We can now apply the two-asset lemma to equation (31) to get

$$
\begin{array}{r}
S_{1}(0) \exp \left(-\frac{\sigma_{1}^{2} T}{2}\right) E_{Q}\left[\exp \left(-Z_{1}\right) I_{\{X<-K\}} I_{\{Y<\tilde{K}\}}\right] \\
=S_{1}(0) \Phi^{2}\left(\hat{x}_{1}, \hat{y}_{1}, \rho_{X Y}\right),
\end{array}
$$

where

$\hat{x}_{1}=\frac{m-K}{\sigma}+\sigma_{1} \rho_{Z_{1} X} \sqrt{T}, \quad \hat{y}_{1}=\tilde{K}+\sigma_{1} \sqrt{T} \rho_{Z_{1} Y}, \quad \rho_{Z_{1} X}=\frac{\sqrt{T}}{\sigma}\left(S_{1}(0) \sigma_{1}-S_{2}(0) \sigma_{2} \rho\right), \quad \rho_{Z_{1} Y}=-\frac{\phi_{1}+\phi_{2} \rho}{\sigma_{\rho}}$,

$\rho_{X Y}=\frac{a-b}{\sigma}, a=S_{2}(0) \frac{\sigma_{2} \sqrt{T}}{\sigma_{\phi}}\left(\phi_{1} \rho+\phi_{2}\right), b=S_{1}(0) \frac{\sigma_{1} \sqrt{T}}{\sigma_{\phi}}\left(\phi_{1}+\phi_{2} \rho\right), \tilde{K}=\frac{\left(\frac{\sigma_{\phi}^{2}}{2}+\phi_{1} \theta_{1}+\phi_{2} \theta_{2}\right) T+\ln \left(\frac{1}{\tilde{a}(\tilde{z})}\right)}{\sigma_{\phi} \sqrt{T}}$

Now consider the second term in equation (30):

$$
S_{2}(0) \exp \left(-\frac{\sigma_{2}^{2} T}{2}\right) E_{Q}\left[\exp \left(\sigma_{2} W_{2}^{Q}(T)\right) I_{\{-\gamma<-K\}} I_{\{\epsilon<\tilde{K}\}}\right]
$$

where

$$
Z_{2}=-\sigma_{2} W_{2}^{Q}(T) \sim N\left(0, \sigma_{1}^{2} T\right), X=-\gamma \sim N\left(-m, \sigma^{2}\right), Y=\frac{\phi_{1} W_{1}^{Q}(T)+\phi_{2} W_{2}^{Q}(T)}{\sigma_{-} \phi \sqrt{T}} \sim N(0,1) .
$$

We need to estimate the correlation coefficients $\rho_{Z_{2} X}$ and $\rho_{Z_{2} Y}$. Proceeding in the same manner as for equation (31), we evaluate the correlation coefficients to be as follows:

$$
\begin{array}{r}
\rho_{Z_{2} X}=\frac{\sqrt{T}}{\sigma}\left(S_{1}(0) \sigma_{1} \rho-S_{2}(0) \sigma_{2}\right), \\
\rho_{Z_{2} Y} \\
=-\frac{\phi_{1} \rho+\phi_{2}}{\sigma_{\phi}} .
\end{array}
$$

Applying the two-asset lemma,

$$
\begin{array}{r}
S_{2}(0) \exp \left(-\frac{\sigma_{2}^{2} T}{2}\right) E_{Q}\left[\exp \left(-Z_{2}\right) I_{\{X<-K\}} I_{\{Y<\tilde{K}\}}\right] \\
=S_{2}(0) \Phi^{2}\left(\hat{x}_{2}, \hat{y}_{2}, \rho_{X Y}\right),
\end{array}
$$


where

$$
\hat{x}_{2}=\frac{m-K}{\sigma}+\sigma_{2} \rho_{Z_{2} X} \sqrt{T}, \hat{y}_{2}=\tilde{K}+\sigma_{2} \sqrt{T} \rho Z_{2 Y}, \rho_{Z_{2} X}=\frac{\sqrt{T}}{\sigma}\left(S_{1}(0) \sigma_{1} \rho-S_{2}(0) \sigma_{2}\right), \rho_{Z_{2} Y}=-\frac{\phi_{1} \rho+\phi_{2}}{\sigma_{\phi}} .
$$

The last term in equation (30) is simply

$$
K E_{Q}\left[I_{\{-\gamma<-K\}} I_{\{\in<\tilde{K}\}}\right]=K \Phi^{2}\left(\hat{x}_{3}, \hat{y}_{3}, \rho_{X Y}\right),
$$

where

$$
\hat{x}_{3}=\frac{m-K}{\sigma}, \hat{y}_{3}=\tilde{K} .
$$

Combining all three terms, equations (32), (34) and (35), we get the stated formula in equation (28). Alternatively, using the BS-approximation:

Theorem 2. Using the BS-approximation for the price of the spread option, the price $p$ of setting up the replicating portfolio at any time $t \leq T$ can be estimated as follows:

$$
p=S_{1}(0) \Phi^{2}\left(\hat{x}_{1}, \hat{y}_{1}, \rho_{3}\right)-S_{2}(0) \Phi^{2}\left(\hat{x}_{2}, \hat{y}_{2}, \rho_{3}\right)-\tilde{z} \Phi^{2}\left(\hat{x}_{3}, \hat{y}_{3}, \rho_{3}\right),
$$

where

$\hat{x}_{1}=\widehat{K}+\sigma_{1} \rho_{1} \sqrt{T}, \quad \hat{y}_{1}=\tilde{K}+\sigma_{1} \rho_{4} \sqrt{T}, \quad \hat{x}_{2}=\widehat{K}+\sigma_{2} \rho_{2} \sqrt{T}, \quad \hat{y}_{2}=\tilde{K}+\sigma_{2} \rho_{5} \sqrt{T}, \quad \hat{x}_{3}=\widehat{K}, \quad \hat{y_{3}}=\tilde{K}$, $\rho_{1}=\frac{\left(\sigma_{1}-\sigma_{2} b \rho\right) \sqrt{T}}{\sqrt{\sigma_{1}^{2} T-2 \sigma_{1} \sigma_{2} b \rho T+\sigma_{2}^{2} b^{2} T}}, \rho_{2}=\frac{\left(\sigma_{1} \rho-\sigma_{2} b\right) \sqrt{T}}{\sqrt{\sigma_{1}^{2} T-2 \sigma_{1} \sigma_{2} b \rho T+\sigma_{2}^{2} b^{2} T}}, \rho_{3}=\frac{\left(\sigma_{2} b \phi_{1} \rho+\sigma_{2} b \phi_{2}-\sigma_{1} \phi_{1}-\sigma_{1} \phi_{2} \rho\right) \sqrt{T}}{\sigma_{\phi} \sqrt{\sigma_{1}^{2} T+\sigma_{2}^{2} b^{2} T-2 \sigma_{1} \sigma_{2} b \rho T}}$, $\rho_{4}=-\frac{\phi_{2} \rho+\phi_{1}}{\sigma_{\phi}}, \quad \rho_{5}=-\frac{\phi_{2}+\phi_{1} \rho}{\sigma_{\phi}}, \quad \sigma_{\phi}=\sqrt{\phi_{1}^{2}+\phi_{2}^{2}+2 \rho \phi_{1} \phi_{2}}, \quad b=\frac{S_{2}(0)}{c}, \quad c=S_{2}(0)+\tilde{z}$, $\tilde{K}=\frac{\left(\frac{\sigma_{\phi}^{2}}{2}+\sigma_{1} \phi_{1}+\sigma_{2} \phi_{2}\right)+\ln \left(\frac{1}{\tilde{a}(\tilde{z})}\right)}{\sigma_{\phi} \sqrt{T}}, \widehat{K}=\frac{\ln \left(\frac{S_{1}(0)}{a(\tilde{z})}\right)-\frac{\sigma_{1}^{2} T}{2}+\frac{\sigma_{2}^{2} b^{2} T}{2}}{\sqrt{\sigma_{1}^{2} T+\sigma_{2}^{2} b^{2} T-2 \rho \sigma_{1} \sigma_{2} b T}}$.

Proof. We need to estimate the following expectation:

$$
E_{Q}\left[\left(S_{1}(T)-S_{2}(T)-K\right) I_{\left\{\frac{d P}{d Q}>\tilde{a}(\tilde{z})\right\}} I_{\left\{S_{1}(T) \geq \frac{c\left(S_{2}(T)\right)^{b}}{E_{Q}\left(\left(S_{2}(T)\right)^{b}\right)}\right\}}\right] .
$$

The first indicator function has already been considered in equation (29); the term in the second indicator function was considered in equation (45) of Appendix C. We can rewrite the above expectation in the following way: 


$$
E_{Q}\left[S_{1}(T) I_{\left\{\epsilon_{1} \leq \tilde{K}\right\}} I_{\left\{\epsilon_{2} \leq \bar{K}\right\}}\right]-E_{Q}\left[S_{2}(T) I_{\left\{\epsilon_{1} \leq \tilde{K}\right\}} I_{\left\{\epsilon_{2} \leq \bar{K}\right\}}\right]-K E_{Q}\left[I_{\left\{\epsilon_{1} \leq \tilde{K}\right\}} I_{\left\{\epsilon_{2} \leq \bar{K}\right\}}\right] .
$$

We can apply the two-asset lemma to each of the three terms in equation (37). Before that, however, we need to estimate the correlation coefficient between $\epsilon_{1}$ and $\epsilon_{2}$,

$$
\begin{gathered}
\rho_{\epsilon_{1} \epsilon_{2}}=E_{Q}\left[\left(\frac{\phi_{1} W_{1}^{Q}(T)+\phi_{2} W_{2}^{Q}(T)}{\sigma_{\phi} \sqrt{T}}\right)\left(\frac{\sigma_{2} b W_{2}^{Q}(T)-\sigma_{1} W_{1}^{Q}(T)}{\sqrt{\sigma_{1}^{2} T-2 \sigma_{1} \sigma_{2} b \rho T+\sigma_{2}^{2} b^{2} T}}\right)\right]= \\
=\frac{\left(\sigma_{2} b \phi_{1} \rho+\sigma_{2} b \phi_{2}-\sigma_{1} \phi_{1}-\sigma_{1} \phi_{2} \rho\right) \sqrt{T}}{\sigma_{\phi} \sqrt{\sigma_{1}^{2} T+\sigma_{2}^{2} b^{2} T-2 \sigma_{1} \sigma_{2} b \rho T}},
\end{gathered}
$$

where we used the fact that

$$
E\left[W^{2}(T)\right]=\operatorname{Var}[W(T)]=T
$$

and

$$
E\left[W_{1}(T) W_{2}(T)\right]=\operatorname{Cov}\left[W_{1}(T) W_{2}(T)\right]=\rho T .
$$

Combining this result with the results of equations (29) and (45), and applying the two-asset lemma to the first term of equation (37),

$$
E_{Q}\left[S_{1}(T) I_{\left\{\epsilon_{1} \leq \tilde{K}\right\}} I_{\left\{\epsilon_{2} \leq \bar{K}\right\}}\right]=S_{1}(0) \Phi^{2}\left(\hat{x}_{1}, \hat{y}_{1}, \rho_{3}\right)
$$

where

$$
\begin{gathered}
\hat{x}_{1}=\tilde{K}+\sigma_{1} \rho_{1} \sqrt{T}, \\
\hat{y}_{1}=\bar{K}+\sigma_{1} \rho_{4} \sqrt{T}, \\
\rho_{1}=\frac{\left(\sigma_{1}-\sigma_{2} b \rho\right) \sqrt{T}}{\sqrt{\sigma_{1}^{2} T-2 \sigma_{1} \sigma_{2} b \rho T+\sigma_{2}^{2} b^{2} T}}, \\
\rho_{3}=\frac{\left(\sigma_{2} b \phi_{1} \rho+\sigma_{2} b \phi_{2}-\sigma_{1} \phi_{1}-\sigma_{1} \phi_{2} \rho\right) \sqrt{T}}{\sigma_{\phi} \sqrt{\sigma_{1}^{2} T+\sigma_{2}^{2} b^{2} T-2 \sigma_{1} \sigma_{2} b \rho T}} \\
\rho_{4}=-\frac{\phi_{2} \rho+\phi_{1}}{\sigma_{\phi}} .
\end{gathered}
$$

The second term of equation (37) evaluates to

$$
E_{Q}\left[S_{2}(T) I_{\left\{\epsilon_{1} \leq \tilde{K}\right\}} I_{\left\{\epsilon_{2} \leq \bar{K}\right\}}\right]=S_{2}(0) \Phi^{2}\left(\hat{x}_{2}, \hat{y}_{2}, \rho_{3}\right),
$$

where 


$$
\begin{aligned}
& \hat{x}_{2}=\tilde{K}+\sigma_{2} \rho_{2} \sqrt{T} \text {, } \\
& \hat{y}_{2}=\bar{K}+\sigma_{2} \rho_{5} \sqrt{T} \text {, } \\
& \rho_{2}=\frac{\left(\sigma_{1} \rho-\sigma_{2} b\right) \sqrt{T}}{\sqrt{\sigma_{1}^{2} T-2 \sigma_{1} \sigma_{2} b \rho T+\sigma_{2}^{2} b^{2} T}}, \\
& \rho_{5} \quad=-\frac{\phi_{2}+\phi_{1} \rho}{\sigma_{\phi}} .
\end{aligned}
$$

The last term of equation (37) is

$$
K E_{Q}\left[I_{\left\{\epsilon_{1} \leq \tilde{K}\right\}} I_{\left\{\epsilon_{2} \leq \bar{K}\right\}}\right]=K \Phi\left(\hat{x}_{3}, \hat{y}_{3}, \rho_{3}\right),
$$

where

$$
\begin{aligned}
& \hat{x}_{3}=\tilde{K}, \\
& \hat{y}_{3}=\bar{K} .
\end{aligned}
$$

Combining equations (38), (39) and (40), we get the formula stated in equation (36).

The existence of closed-form formulas for estimating CvaR-optimal option prices, as per Theorems 1 and 2 , allows constant rebalancing of the replicating portfolio at any moment in time $t \leq T$, which is vital for risk management purposes.

\section{Numerical Illustration and Application to Regulatory Needs}

To see how the methodology would apply to the real market data, we have downloaded the closing price data for Apple Inc. and S\&P500 index from 1st January 2013 to 28th March 2018, with overall 1319 observations. Having transformed the prices to logarithmic returns and having annualised the returns, we obtained the following standard deviations: $\sigma_{1}=0.24, \sigma_{2}=0.12$, where subscript 1 refers

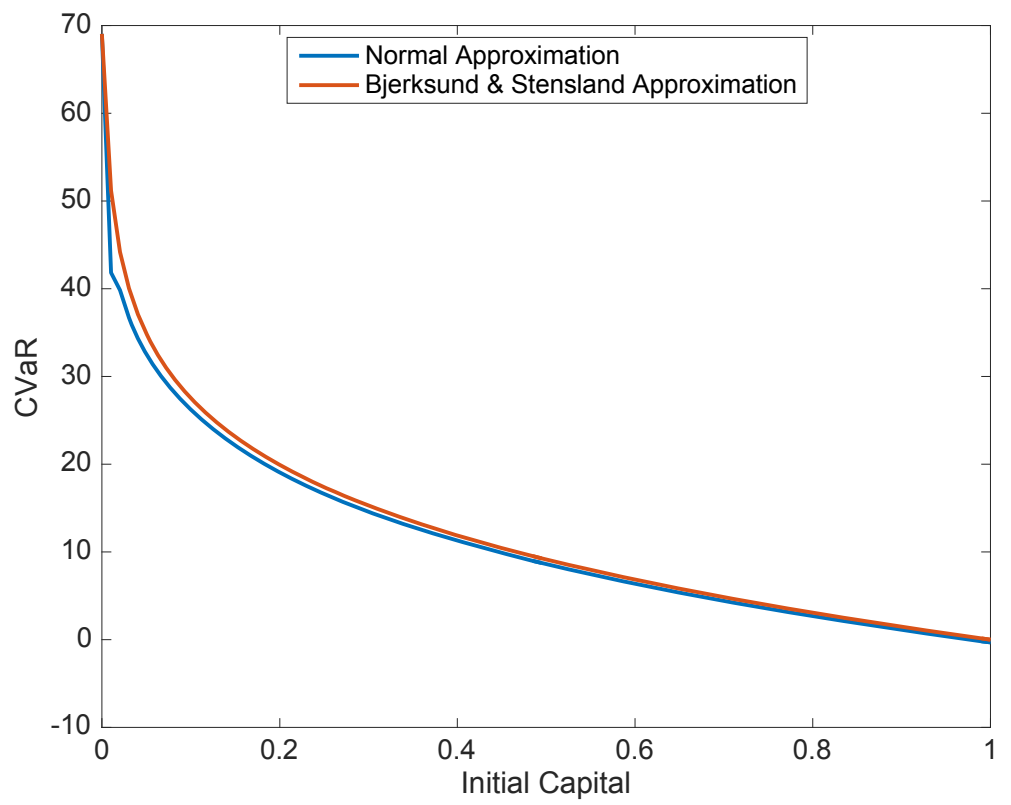

Figure 1. CVaR for varying level of initial capital at $99 \%$ confidence level 
Table 1

CVaR at $99 \%$ confidence level

CVaR

Capital Available, \%
Normal approximation
BS-approximation

\begin{tabular}{lll}
\hline 0 & 68.9700 & 69.0788 \\
10 & 26.0501 & 27.4378 \\
30 & 18.9578 & 19.8209 \\
40 & 14.4591 & 15.1719 \\
51 & 11.1611 & 11.7549 \\
61 & 8.4916 & 9.0196 \\
71 & 6.2363 & 6.7189 \\
81 & 4.2763 & 4.7194 \\
91 & 2.5743 & 2.9413 \\
100 & 0.9873 & 1.3326 \\
\hline
\end{tabular}

Source: The authors.

to Apple Inc. and subscript 2 to S\&P 500 index. The annualised returns are: $\mu_{1}=0.14, \mu_{2}=0.11$. An investor expects to earn a higher rate of return on Apple Inc. to compensate for higher volatility. The estimated correlation coefficient over the period was $\rho=0.5068$. We have standardised the initial prices to be equal

$$
S_{1}(0)=S_{2}(0)=78.4329
$$

The institution has sold an option to exchange a single unit of S\&P500 for the unit of stock of Apple Inc. with an expiration date of one year from now. The price required for complete hedging is determined via equation (6) to be equal to $p=6.49$. We estimate CVaR at $99 \%$. Refer to Fig. 1 , where we plot the level of $C V a R_{0.99}$ for varying levels of the initial capital available as a percentage of the arbitrage-free price.

Table 1 summarises the results of the simulation. We can see that the normal approximation underestimates CVaR for all levels of initial capital available. It is an expected result given that the normal approximation provides lower price estimates when compared with the BS-approximation. We note that both approaches offer only an approximation to the true level of CVaR because there is no exact pricing formula for equation (5).

We can further supplement our analysis by looking at CVaR-efficient portfolios from a regulatory point of view. Suppose that a regulator in the market requires the member institutions to keep a 


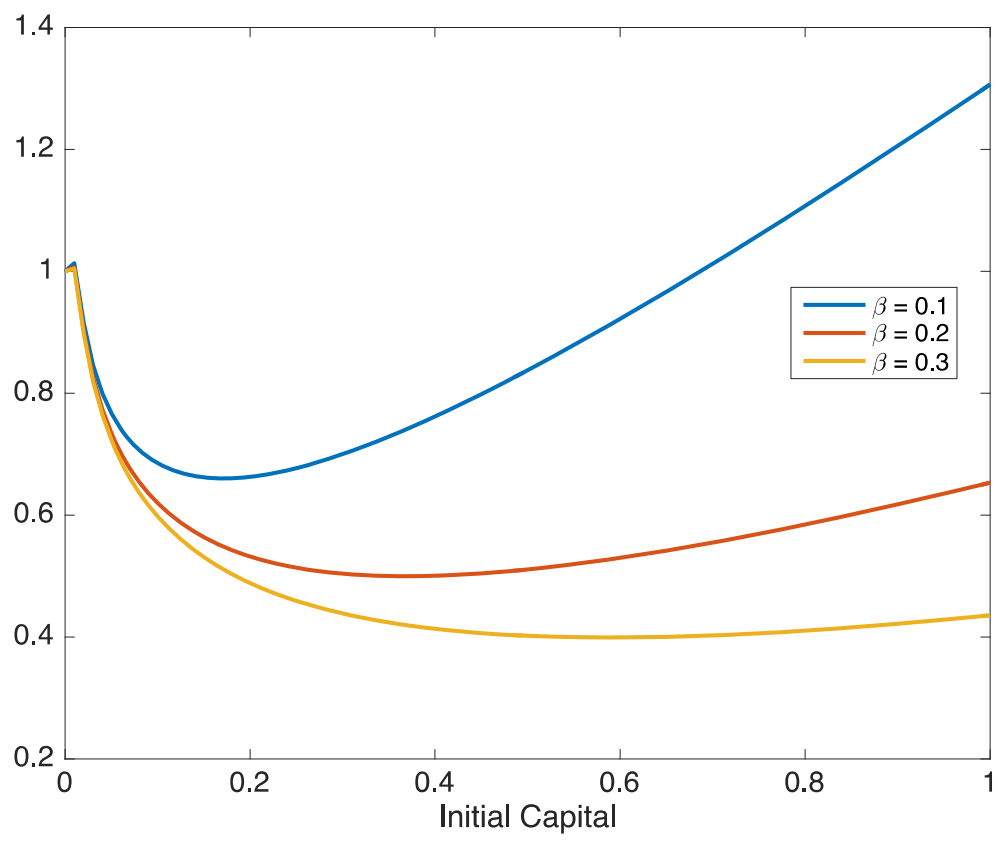

Figure 2. The relative attractiveness of CVaR-efficient portfolio at $99 \%$ confidence level

Source: The authors.

certain amount of capital in reserves depending on the estimated level of CVaR. Let $\beta$ be the necessary amount of capital per unit of CVaR exposure. Denote by

$$
\lambda_{\mathcal{L}}(\widehat{V}(0))=\beta C V a R_{\mathcal{L}}(\widehat{V}(0))+\widehat{V}(0)
$$

the total amount of capital to be kept in reserves provided that the amount of $\widehat{V}(0)$ has been used for hedging purposes at the significance level $\mathcal{L}$. Then the $C V a R_{\mathcal{L}}$ of an unhedged position is $\lambda_{\mathcal{L}}(0)$. Introduce the following ratio:

$$
\Theta_{\mathcal{L}}=\frac{\lambda_{\mathcal{L}}(\widehat{V}(0))}{\lambda_{\mathcal{L}}(0)}
$$

The ratio tells us the relative attractiveness of a CVaR-efficient portfolio. Where $\Theta_{\mathcal{L}}<1$, engaging in CVaR-efficient hedging allows the institution to use less capital to meet the regulatory requirement as compared to an unhedged position and vice versa. We apply this line of analysis to our Apple Inc. and S\&P500 portfolio at a 99\% significance level, and the results we show in Fig. 1.

The above figure clearly indicates that the higher the regulatory requirements, the more attractive a CVaR-efficient portfolio is compared to a portfolio with no hedging. Also, the graph of the relative attractiveness of the CVaR-efficient portfolio as a function of the level of initial capital used is U-shaped, meaning that the relative effectiveness is more sensitive to changes in the capital employed in the tails of the graph. The reader can clearly see this effect from Table 1 . The concavity of the graph in the markets with regulatory requirements means that we can optimise concerning the amount of initial capital to be used to maximise the replicating portfolio's effectiveness.

\section{Conclusion}

In this paper, we have investigated the problem of constructing CVaR-efficient portfolios under capital constraints in the Margrabe market model setting. The two different spread option pricing formulas used provided comparable results. However, neither of the two methods provides an exact solution since no closed form PDF for the difference between two log-normal random variables exists to this moment. 


\section{Acknowledgments}

The research was supported by the NSERC Discovery Grants \#5901 and RES 0043487.

\section{References}

Acerbi, C. (2002). Spectral measures of risk: A coherent representation of subjective risk aversion. Journal of Banking \& Finance, 26(7), 1505-1518.

Artzner, P., Delbaen, F., Eber, J., \& Heath, D. (1999). Coherent measures of risk. Mathematical Finance, 9(3), 203-228.

Basel Committee on Banking Supervision. (2016). Minimum capital requirements for market risk. www.bis.org/ bcbs/publ/d352.pdf (accessed 4th December 2018).

Soubra, A., \& Bastidas-Arteaga, E. (2014). Advanced Reliability Analysis Methods. 10.13140/RG.2.1.1697.7124.

Bjerksund, P., \& Stensland, G. (2006). Closed form spread option valuation. NHH Dept. of Finance \& Management Science Discussion Paper No. 2006/20. https://ssrn.com/abstract=1145206

Browne, S. (1999). Reaching goals by a deadline: Digital options and continuous-time active portfolio management. Advances in Applied Probability, 31(02), 551-577.

Brutti Righi, M., \& Ceretta, P. (2016). Shortfall deviation risk: An alternative for risk measurement. The Journal of Risk, 19(2), 81-116.

Carmona, R., \& Durrleman. (2003). V. Pricing and hedging spread options. SIAM Review, 45(4), 627-685.

Cobb, B., \& Rumi, R. (2012). Approximating the distribution of a sum of log-normal random variables. Sixth European Workshop on Probabilistic Graphical Models, Granada, Spain.

El Karoui, N., \& Quenez, M. (1995). Dynamic programming and pricing of contingent claims in an incomplete market. SIAM Journal on Control and Optimization, 33(1), 29-66.

Fischer, S. (1978). Call option pricing when the exercise price is uncertain, and the valuation of index bonds. The Journal of Finance, 33(1), 169-176.

Foellmer, H., \& Leukert, P. (1999). Quantile hedging. Finance and Stochastics, 3(3), 251-273.

Foellmer, H., \& Leukert, P. (2000). Efficient hedging: Cost versus shortfall risk. Finance and Stochastics, 4(2), $117-146$.

Hcine, M., \& Bouallegue, R. (2015). On the approximation of the sum of lognormals by a log skew normal distribution. International Journal of Computer Networks \& Communications, 7, 135-151.

Kirk, E. (1995). Correlation in the energy markets. In Managing Energy Price Risk, (71-78). London: Risk Publications and Enron Capital \& Trade Resources.

Kulldorff, M. (1993). Optimal control of favorable games with a time limit. SIAM Journal on Control and Optimization, 31(1), 52-69.

Lo, C. F. (2012). The sum and difference of two log-normal random variables. Journal of Applied Mathematics, 2012(Article ID 838397), 1-13. https://doi.org/10.1155/2012/838397

Margrabe, W. (1978). The value of an option to exchange one asset for another. The Journal of Finance, 33(1), $177-186$.

Mehta, N. B., Molisch, A., Wu, J., \& Zhang, J. (2007). Approximating a sum of random variables with a lognormal. IEEE Transactions on Wireless Communications, 6(7), 2690-2699.

Melnikov, A. (2011). Risk Analysis in Finance and Insurance, 2nd ed. Boca Raton, Florida: Chapman \& Hall/CRC.

Melnikov, A., \& Smirnov, I. (2012). Dynamic hedging of conditional value-at-risk. Insurance: Mathematics and Economics, 51(1), 182-190.

Molisch, A. (2013), Wireless Communications. Hoboken, N.J.: Wiley.

Rockafellar, R., \& Uryasev, S. (2000). Optimisation of conditional value-at-risk. The Journal of Risk, 2(3), 21-41.

Shreve, S. (2011). Stochastic Calculus for Finance II. New York: Springer. 


\section{APPENDIXES}

\section{Appendix A Two-asset lemma}

Lemma 1. Let $X \sim N\left(\mu_{x}, \sigma_{x}^{2}\right), Y \sim N\left(\mu_{y}, \sigma_{y}\right)$ and $Z \sim N\left(\mu_{z}, \sigma_{z}^{2}\right)$ be three normally distributed random variables with correlations $\rho_{X Y}, \rho_{X Z}, \rho_{Y Z}$. Then,

$$
E\left[\exp (-Z) I_{\{X<x\}} I_{\{Y<y\}}\right]=\exp \left(-\mu_{z}+\frac{\sigma_{z}^{2}}{2}\right) \Phi^{2}\left(\hat{x}, \hat{y}, \rho_{X Y}\right)
$$

where

$$
\hat{x}=\frac{x-\mu_{x}}{\sigma_{x}}+\sigma_{z} \rho_{X Z}, \hat{y}=\frac{y-\mu_{y}}{\sigma_{y}}+\sigma_{z} \rho_{Y Z}
$$

and $\Phi^{2}$ denotes the two-dimensional normal cumulative distribution function (see Melnikov (2011)).

\section{Appendix B Comparison results for normal approximation and BS-approximation}

Table 2

Spread option: value approximation. The different formulas are from top to bottom: Monte-Carlo simulation, BSapproximation, the normal approximation

\begin{tabular}{|c|c|c|c|c|}
\hline$\sigma_{1}$ & 0.5 & 1 & 3 & 5 \\
\hline \multirow{3}{*}{0.1} & 4.8886 & 6.9092 & 11.937 & 15.372 \\
\hline & 4.885 & 6.9041 & 11.928 & 15.361 \\
\hline & 4.8399 & 6.8061 & 11.404 & 14.228 \\
\hline \multirow{3}{*}{0.15} & 5.1504 & 7.2788 & 12.573 & 16.188 \\
\hline & 5.1447 & 7.2708 & 12.559 & 16.17 \\
\hline & 5.1124 & 7.1577 & 12.103 & 15.121 \\
\hline \multirow{3}{*}{0.2} & 5.7911 & 8.1831 & 14.127 & 18.179 \\
\hline & 5.7833 & 8.172 & 14.108 & 18.153 \\
\hline & 5.7503 & 8.0437 & 13.445 & 16.897 \\
\hline \multirow{3}{*}{0.25} & 6.7008 & 9.4663 & 16.327 & 20.99 \\
\hline & 6.6925 & 9.4544 & 16.305 & 20.959 \\
\hline & 6.6062 & 9.2315 & 15.358 & 18.908 \\
\hline \multirow{3}{*}{0.3} & 7.7852 & 10.994 & 18.937 & 24.313 \\
\hline & 7.7771 & 10.983 & 18.913 & 24.277 \\
\hline & 7.7153 & 10.651 & 17.074 & 20.852 \\
\hline
\end{tabular}

$\mathbf{T}$

Source: The authors. 
Table 3

Spread option: absolute error

\begin{tabular}{|c|c|c|c|c|}
\hline \multirow{2}{*}{$\sigma_{1}$} & \multicolumn{4}{|c|}{$\mathbf{T}$} \\
\hline & 0.5 & 1 & 3 & 5 \\
\hline \multirow{3}{*}{0.1} & 0 & 0 & 0 & 0 \\
\hline & -0.0036 & -0.0051 & -0.009 & -0.011 \\
\hline & -0.0487 & -0.1031 & -0.533 & -1.144 \\
\hline \multirow{3}{*}{0.15} & 0 & 0 & 0 & 0 \\
\hline & -0.0057 & -0.008 & -0.014 & -0.018 \\
\hline & -0.038 & -0.1211 & -0.47 & -1.067 \\
\hline \multirow{3}{*}{0.2} & 0 & 0 & 0 & 0 \\
\hline & -0.0078 & -0.0111 & -0.019 & -0.026 \\
\hline & -0.0408 & -0.1394 & -0.682 & -1.282 \\
\hline \multirow{3}{*}{0.25} & 0 & 0 & 0 & 0 \\
\hline & -0.0083 & -0.0119 & -0.022 & -0.031 \\
\hline & -0.0946 & -0.2348 & -0.969 & -2.082 \\
\hline \multirow{3}{*}{0.3} & 0 & 0 & 0 & 0 \\
\hline & -0.0081 & -0.011 & -0.024 & -0.036 \\
\hline & -0.0699 & -0.343 & -1.863 & -3.461 \\
\hline
\end{tabular}

Source: The authors.

Table 4

Spread option: percentage error

\begin{tabular}{|c|c|c|c|c|}
\hline \multirow{2}{*}{$y$} & \multicolumn{4}{|c|}{$\mathbf{T}$} \\
\hline & 0.5 & 1 & 3 & 5 \\
\hline \multirow{3}{*}{0.1} & $0.00 \%$ & $0.00 \%$ & $0.00 \%$ & $0.00 \%$ \\
\hline & $-0.07 \%$ & $-0.07 \%$ & $-0.08 \%$ & $-0.07 \%$ \\
\hline & $-1.00 \%$ & $-1.49 \%$ & $-4.47 \%$ & $-7.44 \%$ \\
\hline \multirow{3}{*}{0.15} & $0.00 \%$ & $0.00 \%$ & $0.00 \%$ & $0.00 \%$ \\
\hline & $-0.11 \%$ & $-0.11 \%$ & $-0.11 \%$ & $-0.11 \%$ \\
\hline & $-0.74 \%$ & $-1.66 \%$ & $-3.74 \%$ & $-6.59 \%$ \\
\hline \multirow{3}{*}{0.2} & $0.00 \%$ & $0.00 \%$ & $0.00 \%$ & $0.00 \%$ \\
\hline & $-0.13 \%$ & $-0.14 \%$ & $-0.13 \%$ & $-0.14 \%$ \\
\hline & $-0.70 \%$ & $-1.70 \%$ & $-4.83 \%$ & $-7.05 \%$ \\
\hline \multirow{3}{*}{0.25} & $0.00 \%$ & $0.00 \%$ & $0.00 \%$ & $0.00 \%$ \\
\hline & $-0.12 \%$ & $-0.13 \%$ & $-0.13 \%$ & $-0.15 \%$ \\
\hline & $-1.41 \%$ & $-2.48 \%$ & $-5.93 \%$ & $-9.92 \%$ \\
\hline \multirow{3}{*}{0.3} & $0.00 \%$ & $0.00 \%$ & $0.00 \%$ & $0.00 \%$ \\
\hline & $-0.10 \%$ & $-0.10 \%$ & $-0.13 \%$ & $-0.15 \%$ \\
\hline & $-0.90 \%$ & $-3.12 \%$ & $-9.84 \%$ & $-14.24 \%$ \\
\hline
\end{tabular}

Source: The authors. 


\section{Appendix C BS-approximation}

Consider the following expression:

$$
\begin{aligned}
& E_{Q}\left[\left(S_{1}(T)-S_{2}(T)-K\right) I_{\left\{S_{1}(T) \geq \frac{c\left(S_{2}(T)\right)^{b}}{E_{Q}\left(\left(S_{2}(T)\right)^{b}\right)}\right\}}\right] \\
& =E_{Q}\left[S_{1}(T) I_{\left\{S_{1}(T) \geq \frac{c\left(S_{2}(T)\right)^{b}}{E_{Q}\left(\left(S_{2}(T)\right)^{b}\right)}\right\}}\right]-E_{Q}\left[S_{2}(T) I_{\left\{S_{1}(T) \geq \frac{c\left(S_{2}(T)\right)^{b}}{E_{Q}\left(\left(S_{2}(T)\right)^{b}\right)}\right\}}\right] \\
& -E_{Q}\left[K I_{\left\{S_{1}(T) \geq \frac{c\left(S_{2}(T)\right)^{b}}{E_{Q}\left(\left(S_{2}(T)\right)^{b}\right)}\right\}}\right] \text {. }
\end{aligned}
$$

The term in the denominator is

$$
E_{Q}\left(\left(S_{2}(T)\right)^{b}\right)=\left(S_{2}(0)\right)^{b} \exp \left(-\frac{\sigma_{2}^{2} b T}{2}\right) E_{Q}\left[\exp \left(\sigma_{2} b W_{2}^{Q}(T)\right)\right]=\left(S_{2}(0)\right)^{b} \exp \left(\frac{\sigma_{2}^{2} b(b-1) T}{2}\right)
$$

Let us now simplify the term in the indicator function:

$$
\begin{gathered}
S_{1}(T) \geq \frac{c\left(S_{2}(T)\right)^{b}}{E_{Q}\left(\left(S_{2}(T)\right)^{b}\right)}, S_{1}(0) \exp \left(-\frac{\sigma_{1}^{2} T}{2}+\sigma_{1} W_{1}^{Q}(T)\right) \geq c \exp \left(-\frac{\sigma_{2}^{2} b^{2} T}{2}+\sigma_{2} b W_{2}^{Q}(T)\right) \\
\ln \left(\frac{S_{1}(0)}{c}\right)-\frac{\sigma_{1}^{2} T}{2}+\frac{\sigma_{2}^{2} b^{2} T}{2} \geq \sigma_{2} b W_{2}^{Q}(T)-\sigma_{1} W_{1}^{Q}(T) .
\end{gathered}
$$

Since

$\sigma_{2} b W_{2}^{Q}(T)-\sigma_{1} W_{1}^{Q}(T) \sim N\left(0, \sigma_{1}^{2} T-2 \sigma_{1} \sigma_{2} b \rho T+\sigma_{2}^{2} b^{2} T\right)$,

the inequality in equation (45) is equivalent to $\in \leq d_{3}$,

where

$$
\in=\frac{\sigma_{2} b W_{2}^{Q}(T)-\sigma_{1} W_{1}^{Q}(T)}{\sqrt{\sigma_{1}^{2} T-2 \sigma_{1} \sigma_{2} b \rho T+\sigma_{2}^{2} b^{2} T}} \sim N(0,1), d_{3}=\frac{\ln \left(\frac{S_{1}(0)}{c}\right)-\frac{\sigma_{1}^{2} T}{2}+\frac{\sigma_{2}^{2} b^{2} T}{2}}{\sqrt{\sigma_{1}^{2} T-2 \sigma_{1} \sigma_{2} b \rho T+\sigma_{2}^{2} b^{2} T}} .
$$

Consider the first term in the original expectation, i.e. equation (44), 


$$
E_{Q}\left[S_{1}(T) I_{\left\{S_{1}(T) \geq \frac{c\left(S_{2}(T)\right)^{b}}{\left.\left.E_{Q}\left(S_{2}(T)\right)^{b}\right)\right\}}\right]}\right]=S_{1}(0) \exp \left(-\frac{\sigma_{1}^{2} T}{2}\right) E_{Q}\left[\exp \left(\sigma_{1} W_{1}^{Q}(T)\right) I_{\left\{\in \leq d_{3}\right\}}\right]
$$

Applying the two-asset lemma to the expectation term,

$$
E_{Q}\left[\exp \left(\sigma_{1} W_{1}^{Q}(T)\right) I_{\left\{\in \leq d_{3}\right\}}\right]=\exp \left(\frac{\sigma_{1}^{2} T}{2}\right) \Phi\left(d_{3}+\frac{\left(\sigma_{1}-\sigma_{2} b \rho\right) \sqrt{T}}{\sqrt{\sigma_{1}^{2} T-2 \sigma_{1} \sigma_{2} b \rho T+\sigma_{2}^{2} b^{2} T}}\right)
$$

which leads to

$$
E_{Q}\left[S_{1}(T) I_{\left\{S_{1}(T) \geq \frac{c\left(S_{2}(T)\right)^{b}}{E_{Q}\left(\left(S_{2}(T)\right)^{b}\right)}\right\}}\right]=S_{1}(0) \Phi\left(d_{1}\right)
$$

where

$$
d_{1}=d_{3}+\sigma_{1} \rho_{1} \sqrt{T}, \rho_{1}=\frac{\left(\sigma_{1}-\sigma_{2} b \rho\right) \sqrt{T}}{\sqrt{\sigma_{1}^{2} T-2 \sigma_{1} \sigma_{2} b \rho T+\sigma_{2}^{2} b^{2} T}} .
$$

Let us consider the second term of equation (44),

$$
E_{Q}\left[S_{2}(T) I_{\left\{S_{1}(T) \geq \frac{c\left(S_{2}(T)\right)^{b}}{E_{Q}\left(\left(S_{2}(T)\right)^{b}\right)}\right\}}\right]=S_{2}(0) \exp \left(-\frac{\sigma_{2}^{2} T}{2}\right) E_{Q}\left[\exp \left(\sigma_{2} W_{2}^{Q}(T)\right) I_{\left\{\in \leq d_{3}\right\}}\right] .
$$

Applying the two-asset lemma again, we have

$$
E_{Q}\left[S_{2}(T) I_{\left\{S_{1}(T) \geq \frac{c S_{2}^{b}(T)}{E_{Q}\left(S_{2}^{b}(T)\right)}\right\}}\right]=S_{2}(0) \Phi\left(d_{2}\right)
$$

where

$$
d_{2}=d_{3}+\sigma_{2} \rho_{2} \sqrt{T}, \rho_{2}=\frac{\left(\sigma_{1} \rho-\sigma_{2} b\right) \sqrt{T}}{\sqrt{\sigma_{1}^{2} T-2 \sigma_{1} \sigma_{2} b \rho T+\sigma_{2}^{2} b^{2} T}} .
$$


Finally, the third term of equation (44) is simply

$$
E_{Q}\left[K I_{\left\{S_{1}(T) \geq \frac{c\left(S_{2}(T)\right)^{b}}{\left.E_{Q}\left(S_{2}(T)\right)^{b}\right)}\right\}}\right]=K \Phi\left(d_{3}\right) .
$$

Combining those three term, we get the BS-approximation as in equation (11).

\section{ABOUT THE AUTHORS / ИНФОРМАЦИЯ ОБ АВТОРАХ}

Chingis Maksimov - Canadian Western Bank, Edmonton, AB, Canada

chingis@ualberta.ca

Чингис Максимов - Canadian Western Bank, Эдмонтон, АВ, Канада

Alexander Melnikov - Professor, DSc from Steklov Mathematical Institute, Russia; PhD from Steklov Mathematical Institute, Russia; MSc from Moscow State University, Russia. University of Alberta, Edmonton, $\mathrm{AB}$, Canada

melnikov@ualberta.ca

Александр Мельников - профессор, доктор физико-математических наук. Степень кандидата и доктора наук получил в Математическом институте им. В.А. Стеклова РАН. Выпускник московского государственного университета им. М.В. Ломоносова. Университет Альберты, Эдмонтон, АВ, Канада

The article was submitted on 17.05.2021, reviewed on 16.07.2021, and accepted for publication on 17.08.2021.

The authors read and approved the final version of the manuscript.

Статья поступила в редакцию 17.05.2021; после рецензирования 16.07.2021; принята к публикации 17.08.2021. Авторы прочитали и одобрили окончательный вариант рукописи. 